ratio correlated significantly with $\mathrm{BMI}$ in both cohorts $\left(\mathrm{r}^{2}=0.19\right.$ for study cohort, $r^{2}=0.25$ for validation cohort; $\left.p<0.0001\right)$. MUST score had only moderate value in predicting weight loss in the study cohort (AUC $0.7 ; 95 \% \mathrm{Cl}$ : 0.58-0.82). Specifically, $46.5 \%$ of SSc patients lost $>10 \%$ wt despite having "no" or "mild" MUST scores. Logistic regression analysis identified the combination of BMI and A/L as the best PREdictor of MAlnutrition in Systemic Sclerosis (PREMASS). The formula $12.18-\left(0.63^{*} \mathrm{BMI}\right)+\left(1.51^{*} \mathrm{~A} / \mathrm{L}\right)$ predicted the end point with $\mathrm{AUC}=0.91(95 \%$ Cl:0.77-0.84). A PREMASS score $>0.23$ showed $91.3 \%$ sensitivity (95\% Cl:79.79-100) and $80.46 \%$ specificity (95\% Cl:72.13-88.79) for $>10 \%$ wt loss with an overall $55.26 \%$ positive predictive value (PPV) $(95 \%$ Cl:39.45-71.07) and $97.22 \%$ negative predictive value (NPV) (95\% Cl:93.43-100) and a relative risk (RR) of 19.90 (95\% Cl:4.93-80.37). In the validation cohort, PREMASS showed $76.47 \%$ sensitivity (95\% Cl:56.31-96.63) and $75.47 \%$ specificity (95\% Cl:63.89-87.06) with an overall 50\% PPV (95\% Cl:30.78-69.22) and 90.91\% NPV (95\% Cl:82.41-99.4) and a RR of 5.5 (95\% Cl:2-15.10).

Conclusions: PREMASS is the first validated index for weight loss risk stratification in the following 12 months in SSc. Prediction of future weight loss in SSc could aid both in clinical management and stratification/enrichment in clinical trials.

Disclosure of Interest: None declared

DOI: 10.1136/annrheumdis-2018-eular.4102

\section{THU0394 ENTHESITIS IN SYSTEMIC SCLEROSIS (SSC): AN ULTRASOUND (US) PILOT STUDY}

${ }^{1}$ R. Terenzi, R. Karalilova ${ }^{2}$, S. Bellando-Randone ${ }^{1}$, S. Guiducci ${ }^{1}$, M. Manetti ${ }^{3}$, E. Romano ${ }^{1}$, D.E. Furst ${ }^{1,4,5}$, G. Pacini ${ }^{1}$, A. Batalov ${ }^{2}$, M. Matucci-Cerinic ${ }^{1}$

${ }^{1}$ Department Of Medicine, Division Of Rheumatology, UNIVERSITÀ DEGLI STUDI DI FIRENZE, Firenze, Italy, ${ }^{2}$ Clinic of Rheumatology, Medical University of Plovdiv, Plovdiv, Bulgaria; ${ }^{3}$ Department Of Medicine, Division Of Anatomy and Histology, UNIVERSITÀ DEGLI STUDI DI FIRENZE, Firenze, Italy; ${ }^{4}$ Department Of Medicine, Division Of Rheumatology, University of California, Los Angeles, California; ${ }^{5}$ University of Washington, Seattle, Washington, USA

Background: Articular involvement is frequently encountered in SSc and previous US studies suggest that synovitis is the commonest manifestation. Recently, it has been reported that SSc patients may show typical "hall-marks" of spondyloarthritis (SpA). Apart from tendon involvement which is a common event, sacroiliitis has been estimated to have a prevalence of $23 \%$.

Objectives: To estimate the prevalence of entheseal and Synovio-Entheseal Complex (SEC) modifications in SSC

Methods: 30 SSc patients (2013 ACR/EULAR classification criteria) without a history of articular involvement (4 male, 26 female, mean age $53,3 \pm 16,6$ years) were included in this pilot cross sectional US study. 12 healthy subjects ( 2 male, 10 female, mean age $46,9 \pm 5,8$ years) were used as controls. The entheseal sites were the lateral epicondylar common extensor tendons (CET), and sites of the Glasgow Ultrasound Enthesis Scoring System. The GUESS score was also calculated as a soft tissue score (GUESS soft tissue) and as a bone score (GUESS bone). US assessment was performed with a MyLab 70XVG scanner equipped with a 6-18 MHz linear transducer (Esaote). Only the epicondylar region was evaluated with PowerDoppler US (PDUS), using semi-quantitative graded from 0 to 3. Involvement of SEC was evaluated at the epicondylar region in SSc patients who presented a PDUS signals $\geq 1$ in the CET closer than $2 \mathrm{~mm}$ from the bony surface. SEC involvement was defined as the presence of a PDUS signal $\geq 2$ in the elbow epicondylar synovial fold proximal to the annular ligament $(A L)$, inferior to the bone insertion of CET and to the radial collateral ligament (RCL). Statistical analyses were carried out using Mann-Whitney $\mathrm{U}$, Spearman correlation and Chisquare tests. Results were considered significant if $p<0,05$

Results: In SSc, the GUESS and GUESS soft tissue scores were significantly higher $(5.67 \pm 0.87$ and $3.43 \pm 0.44$, respectively) than in controls $(1.25 \pm 0.41$ and $0.92 \pm 0.29$, respectively) $(p<0,0001)$ as was the GUESS bone score (SSc 2.23 \pm 0.55 vs controls $0.33 \pm 0.22 ; p<0,05$ ). The CET entheses of $S S c$ patients showed significantly more US B-mode alterations than controls (hypoechogenicity $\chi^{2}=5.95, p=0.015$, cortical irregularity $\chi^{2}=7.90, p=0.005$, calcification/enthesophytes $\left.\chi^{2}=3.78, p=0.05\right)$. A PD signal in the CET enthesis was found in $18 / 60$ sites in SSc and in 1/24 in controls. The PD signal of the CET enthesis was significantly higher in SSc patients than in healthy controls $(0.47 \pm 0.10$ and $0.08 \pm 0.08$ respecctively, $p=0.018)$ as was the presence of SEC inflammation $\left(\chi^{2}=4.54, p=0.033\right)$. In $\mathrm{SSc}$, there was a strong correlation between the presence of PD signal at CET entheses and concomitant SEC inflammation ( $r h o=0.655, p<0.0001$ ) but there were no correlations between GUESS score or CET enthesitis and disease subset, disease duration, antibodies, DLCO, FVC, DLCO/VA.

Conclusions: Our data show that SSc patients frequently present the usual US features of enthesitis. Moreover, CET enthesitis were correlated with SEC inflammation suggesting that entheseal inflammation in SSc may share same microanatomical targets as SpA.

Disclosure of Interest: None declared

DOI: 10.1136/annrheumdis-2018-eular.4174

\section{THU0395 \\ INFLUENCE OF SETTING AN UPPER LIMIT OF THE MRSS AS AN INCLUSION CRITERION IN SSC CLINICAL TRIALS ON THE RATIO OF SKIN FIBROSIS PROGRESSION VS. IMPROVEMENT - AN ANALYSIS OF THE GENISOS COHORT}

${ }^{1,2}$ C. Mihai, R. Dobrota ${ }^{1,2}$, S. Assassi ${ }^{3}$, M. Mayes ${ }^{3}$, O. Distler ${ }^{1}$, on behalf of GENISOS COLLABORATION. ${ }^{1}$ Department of Rheumatology, Zurich University Hospital, Zurich, Switzerland; ${ }^{2}$ Internal Medicine and Rheumatology, Cantacuzino Hospital, Carol Davila University of Medicine and Pharmacy, Bucharest, Romania; ${ }^{3}$ Division of Rheumatology, The University of Texas Health Science Center at Houston, Houston, TX, USA

Background: Skin involvement is a main domain in the assessment of patients with systemic sclerosis (SSc), and the modified Rodnan skin score (mRSS) is a primary outcome measurement in SSc clinical trials. Recent studies on large SSc cohorts have shown that lowering the upper threshold of mRSS as a study inclusion criterion leads to cohort enrichment with patients with progressive skin disease. Limitations of these studies were lack of racial diversity and low proportion of patients with anti-RNA-Polymerase III (Pol3) antibodies.

Objectives: As the Texas-based GENISOS is an ethnically diverse cohort and includes a large proportion of Pol3-positive patients, this study aimed to assess the effect of different mRSS cut-off values at baseline on progression of skin fibrosis after one year of follow-up.

Methods: We extracted data from GENISOS for patients fulfilling the $1980 \mathrm{ACR}$ criteria for SSc and the Le Roy criteria for diffuse cutaneous SSc, who had a mRSS $\geq 7$ at inclusion and a follow-up visit with documented mRSS at $12 \pm 2$ months. Progressors were defined as having an increase in mRSS $>5$ points and $\geq 25 \%$ from the baseline to 2 nd visit, while regressors were defined as having a decrease in mRSS of $>5$ points and $\geq 25 \%$. To identify the optimal cut-off of baseline mRSS that yields the highest sensitivity for progressive skin fibrosis, we developed ROC curves and logistic regression models with "progression" as outcome variable and a binary variable of baseline mRSS cut-off point as predictor. Results: We identified 152 patients (age and disease duration [median, Q1-Q3, years] 49.5, 40.2-57.3 and 2.2, 1.1-3.3 respectively, 22.4\% males) who matched the inclusion criteria. The proportion of patients of African American ethnicity was $31 / 152$ and 50/152 were Pol3-positive patients, both substantially higher than in European cohorts.

After one year, 17 patients (11.2\%) classified as progressors and 51 (33.6\%) as regressors. Progressors were more frequently positive for anti-topoisomerase antibodies (37.5\% vs. $15.3 \%, p=0.028)$, negative for anti-Pol3 antibodies $(93.8 \%$ vs. $62.3 \%, p<0.012$ ), had a shorter disease duration (median, Q1-Q3: $1.3,0.5-2.2$ vs. 2.4, 1.1-3.5 years, $p<0.005$ ) and lower mRSS (median, Q1-Q3: 21, 11-25 vs $24,16-31, p<0.012$ ) than non-progressors.

Sixteen of 17 progressors, but only 33 of 51 regressors had a baseline mRSS $\leq 27$. The mRSS cut-off of $\leq 27$ had the highest probability of progression (odds ratio 9.1, 95\% confidence interval 1.2-70.9, p<0.035, area under the curve 0.652). Using this cut-off as an inclusion criterion (vs. no cut-off) would have included $94 \%$ of all progressors, but only $65 \%$ of all regressors and $67 \%$ of all patients. The figure 1 displays absolute numbers of progressors and regressors at 1 year for each mRSS cut-off.

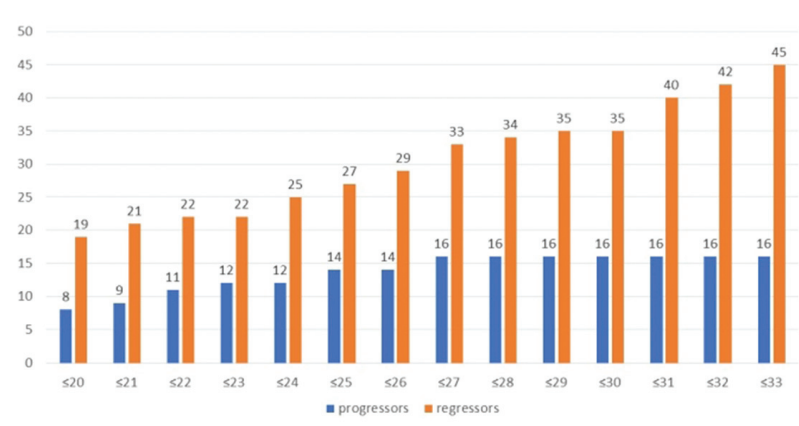

Conclusions: This analysis reconfirmed, in a population rich in patients of African American origin and with high prevalence of Pol3 antibodies, that setting a lowe upper threshold of mRSS at study inclusion increases the proportion of progressors and reduces the absolute number of regressors. This confirms that this recruitment strategy should be used for clinical trial design in early diffuse SSc.

Acknowledgements: The authors wish to thank all GENISOS investigators and patients.

Disclosure of Interest: C. Mihai Grant/research support from: Actelion Pharmaceuticals Ltd, Abbvie, Speakers bureau: Roche, Geneva Romfarm, R. Dobrota Grant/research support from: Actelion Pharmaceuticals Ltd, Pfizer, S. Assassi Grant/research support from: U.S. National Institutes of Health-National Institute of Arthritis and Musculoskeletal and Skin Diseases, U.S. DOD Peer Reviewed 